\title{
2014 Steele Prizes
}

The 2014 AMS Leroy P. Steele Prizes were presented at the 120th Annual Meeting of the AMS in Baltimore, Maryland, in January 2014. The Steele Prizes were awarded to LuIS A. CAFFARELLI, ROBERT KOHN, and LOUIS NIRENBERG for Seminal Contribution to Research; DMITRI Y. BURAGO, YURI D. BURAGO, and SERGEI V. IVANOV for Mathematical Exposition; and PHILLIP A. GRIFFITHS for Lifetime Achievement.

\section{Seminal Contribution to Research: Luis A. Caffarelli, Robert Kohn, and Louis Nirenberg}

\section{Citation}

The 2014 Leroy P. Steele Prize for Seminal Contribution to Research is awarded to Luis A. Caffarelli, Robert Kohn, and Louis Nirenberg for their paper "Partial Regularity for Suitable Weak Solutions of the Navier-Stokes Equation", Communications on Pure and Applied Mathematics 35 (1982), no. 6, 771-831. This paper was and remains a landmark in the understanding of the behavior of solutions to the Navier-Stokes equations and has been a source of inspiration for a generation of mathematicians.

The Navier-Stokes equations are fundamental to the mathematical understanding of fluid dynamics. The pioneering works of Leray and later Hopf established the existence, globally in time, of the Leray-Hopf weak solutions. The arguments of Leray and Hopf left open the possibility that these weak solutions fail to be smooth at a rather sparse closed set of times (of finite 1/2-Hausdorff dimension in time) where uniqueness can potentially be lost.

The work of Caffarelli, Kohn, and Nirenberg was a huge leap forward on this notoriously difficult problem. Building on work of V. Scheffer, Caffarelli, Kohn, and Nirenberg greatly improved Scheffer's results and earlier work of others on the Navier-Stokes equation. They proved the existence of so-called suitable weak solutions as well as their partial regularity. They obtain constraints on the size of the singular set in space and time. The singular set has zero measure with respect to a natural one-dimensional Hausdorff measure defined using a parabolic notion of distance. One important consequence is that any two singular points can be separated by a space-time cylinder on the boundaries of which the solution is regular.

DOI: http://dx.doi.org/10.1090/noti1099
The paper has been a kind of textbook for a whole generation of Navier-Stokes researchers, motivating many of the later developments and simplifications. Though a solution to the global existence of smooth solutions of the Cauchy problems remains open and is now one of the Clay Millennium Problems, the partial regularity theorem of Caffarelli, Kohn, and Nirenberg remains among the sharpest results in this direction.

\section{Biographical Sketches}

Luis A. Caffarelli obtained his Master's of Science (1969) and Ph.D. (1972) at the University of Buenos Aires. Since 1996, he has held the Sid Richardson Chair in Mathematics at the Univer-

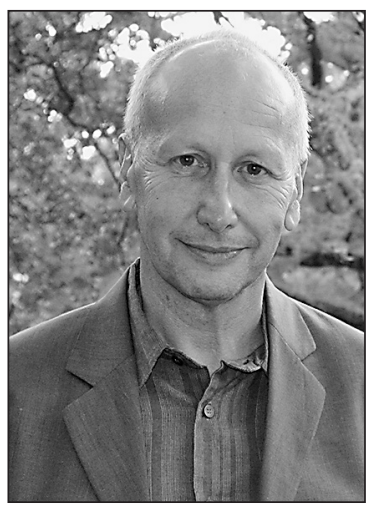

Luis A. Caffarelli

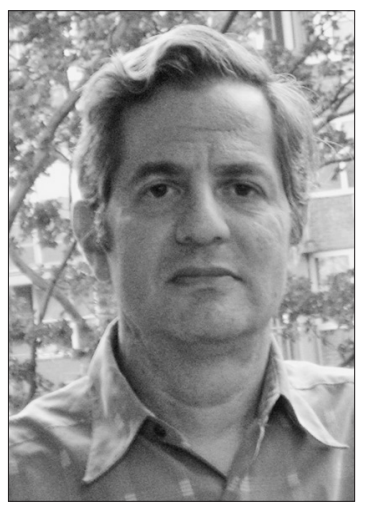

Robert Kohn sity of Texas at Austin. Some of his most significant contributions are the regularity of free boundary problems and solutions to nonlinear elliptic partial differential equations, optimal transportation theory, and, more recently, results in the theory of homogenization.

In 1991 he was elected to the National Academy of Sciences. He received the Bôcher Memorial Prize in 1984. He also received the prestigious 2005 Rolf Schock Prize in Mathematics of the Royal Swedish Academy of Sciences, the 2009 Leroy P. Steele Prize for Lifetime Achievement in Mathematics, and the 2012 Wolf Prize.

Robert Vita Kohn graduated from Harvard University in 1974. He got his M.Sc. at the University of Warwick in 1975, then went to Princeton, where he got his Ph.D. in 1979 under the guidance of Fred Almgren. From 1979-1981 he was a visiting member at New York University's Courant Institute of Mathematical Sciences, supported by an NSF Mathematical Sciences Postdoctoral Fellowship. In 1981 he joined the faculty of the Courant Institute, and 


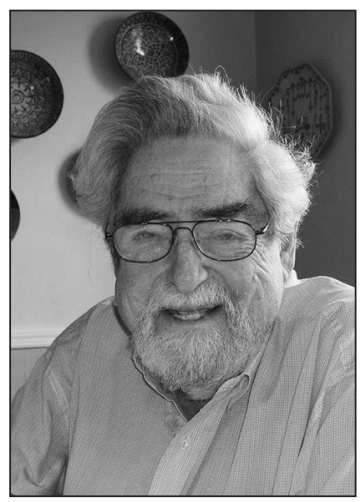

Louis Nirenberg he has remained there since. He has been professor of mathematics at NYU since 1988.

Besides the NavierStokes equations, he has worked on many PDE problems, including electric impedance tomography, relaxation of variational problems, blowup of semilinear heat equations, and homogenization. In recent years much of his research has addressed problems from materials science, with particular emphasis on energy-driven pattern formation. His recognitions include an Alfred P. Sloan Foundation Fellowship (1984-1986) and SIAM's Ralph E. Kleinman Prize (1999). He was a Plenary Speaker at ICM 2006 Madrid and an Invited Speaker at ICIAM 2007, and he is both a Fellow of the American Mathematical Society and a SIAM Fellow.

Louis Nirenberg, professor emeritus at Courant Institute, New York University, received his B.Sc. at McGill University in 1945 and his Ph.D. at New York University in 1949. His entire professional career has been at NYU. His research interests are in mathematical analysis, in particular, partial differential equations, complex analysis, differential geometry, and fluid flow. He also has a deep interest in music and cinema.

\section{Joint Response from Luis A. Caffarelli, Robert Kohn, and Louis Nirenberg}

It is a great honor to receive this award. The Navier-Stokes equation was originally proposed to describe incompressible viscous flows in moderate regimes. Its study has led over the years to many fundamental and challenging problems, which have motivated the development of deep ideas in many areas of analysis and applied mathematics. Any work on it relies strongly on the deep contributions of many who came before.

In this regard, we particularly want to thank and recognize Vladimir Scheffer for his fundamental contributions to this area. Scheffer was the first to apply partial regularity methods to three-dimensional Navier-Stokes-asking, around 1976, what can be said about the size of the singular set. His papers on partial regularity drew our attention to the problem, and his ideas were crucial to our analysis.

Many of the central issues concerning NavierStokes are still open. Deep work continues to be motivated by challenges such as the regularity and uniqueness of solutions, the relationship to turbulence, and the Euler equations, etc.
We did this work at a time when the three of us were at the Courant Institute, benefitting from its wonderful atmosphere. We would like to thank our colleagues at the time for their support.

Finally, we thank the Steele Prize Committee and the American Mathematical Society for choosing us for this award.

\section{Mathematical Exposition: Dmitri Y. Burago, Yuri D. Burago, and Sergei V. Ivanov}

\section{Citation}

The 2014 Leroy P. Steele Prize for Mathematical Exposition is awarded to Dmitri Y. Burago, Yuri

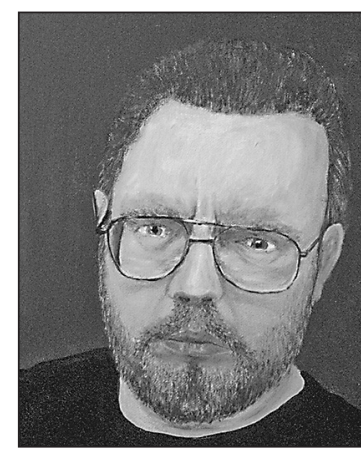

Dmitri Y. Burago

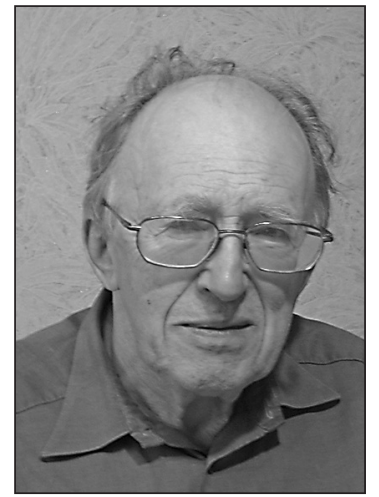

Yuri D. Burago

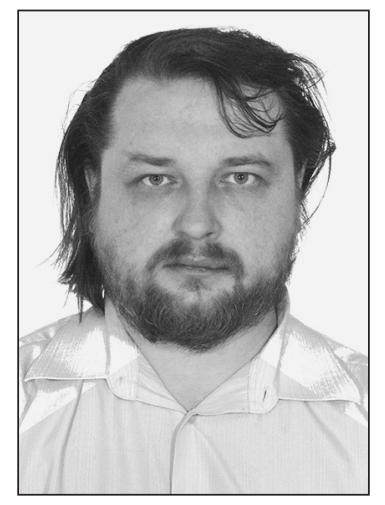

Sergei Ivanov
D. Burago, and Sergei A. Ivanov for the book A Course in Metric Geometry, Graduate Studies in Mathematics, 33, American Mathematical Society, Providence, RI, 2001, in recognition of excellence in exposition and promotion of fruitful ideas in geometry.

The publishing of this book made available to the mathematical community the emerging ideas and methods of synthetic geometry, initiated by Alexandrov and Gromov. These ideas provided a completely new approach to differential geometry replacing the traditional heavy analytic machinery by a description based on easily accessible, simple geometric axioms that have an immediate appeal to geometric intuition.

An influential contribution through the years, this book provided fundamental tools in connection with geodesically convex spaces, optimal transportation in Alexandrov spaces with curvature bounded below, and has been widely referred to recently in connection to the solution of the Geometrization Conjecture.

This book has clearly left a visible imprint on the landscape of today's geometry. It provides great help to orient students in the introductory 
studies of synthetic methods and to guide young geometers in their research.

\section{Biographical Sketches}

Dmitri (Dima) Burago, a Distinguished Professor at the Pennsylvania State University, received his degree from Leningrad (now St. Petersburg) State University. He moved to the U.S. about twenty years ago. Before that, he was doing lots of crazy things: mathematics, whitewater kayaking, a small zoo at home, kickboxing, and much more. He was teaching school kids and is very proud of his students. He had fantastic teachers, and all he has done in his life is due to their input. In the U.S., he continues doing strange things, including mathematics, Russian literature, painting, and of course, teaching. He has received a prize of the St. Petersburg Mathematical Society and has a Faculty Medal from Penn State. He spoke at the 1998 ICM in Berlin.

Yuri Burago was born in St. Petersburg (formerly Leningrad), Russia, in 1936. He graduated from St. Petersburg State University in 1959 and received his Ph.D. in 1961. His advisers were A. D. Alexandrov and V. A. Zalgaller. Yuri Burago defended his doctoral thesis (the second degree in Russia) in 1969. From 1962 to the present he has been the head of the geometry and topology laboratory. Further, he is a full professor (half position) at St. Petersburg State University. Among Yuri Burago's students are Sergei Buyalo, Grisha Perelman, and Anton Petrunin. Yuri Burago worked in a variety of areas of geometry that include so-called 2-manifolds of bounded curvature, irregular surfaces in Euclidean spaces, Riemannian geometry in the large, Alexandrov spaces, and even the theory of functions in irregular domains. He wrote several books, including Introduction to Riemannian Geometry (only in Russian) and Geometric Inequalities, both jointly with Victor Zalgaller.

Sergei Ivanov was born in St. Petersburg, Russia, in 1972. He received his Ph.D. from St. Petersburg State University in 1996. He spent most of his scientific career at St. Petersburg Department of Steklov Mathematical Institute, where he is currently a principal research fellow. He combines this position with teaching at the Mathematics and Mechanics Department of St. Petersburg State University. He was an invited speaker at the ICM (Hyderabad 2010) and was elected as a corresponding member of the Russian Academy of Sciences in 2011.

\section{Joint Response from Dima Burago, Yuri Burago, and Sergei Ivanov}

We are honored and grateful. There are many other books that deserve the prize, so the fact that we are selected makes us humble and speechless.

It took us more than three years to complete the work, and it would take forever without support and encouragement from our colleagues and AMS editors. We are grateful to all the colleagues who supported us and to the people who taught us.

We ourselves represent three generations of Russian geometric tradition: Dmitri's first and primary teacher was Yuri, and in his turn Dmitri taught Sergei.

Our aim when writing the book was to try to bridge the gap between students and the existing literature on the subject. In particular, we kept in mind some of Gromov's works as "bridge destinations". We are happy to know that the book turned out to be useful to many students and researchers.

The list of people without whom this work would be impossible is perhaps too long for this response, especially since there are three of us. We just want to thank all of them!

\section{Lifetime Achievement: Phillip A. Griffiths}

\section{Citation}

The 2014 Leroy Steele Prize for Lifetime Achievement is awarded to Phillip A. Griffiths for his

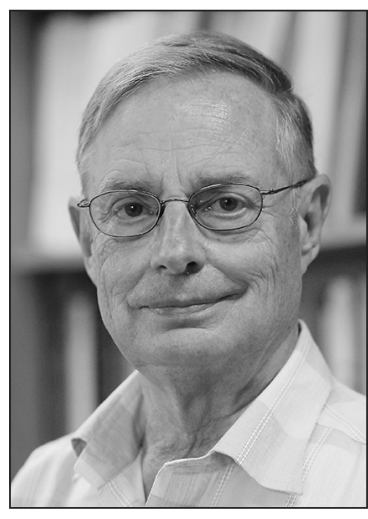

Phillip A. Griffiths contributions to our fundamental knowledge in mathematics, particularly algebraic geometry, differential geometry, and differential equations.

It would not be possible in the space of this column to give a detailed description of all of the areas in which Phillip A. Griffiths has made essential and fundamental contributions in mathematics.

Griffiths' work in algebraic geometry has inspired at least two generations of leading mathematicians working in this area, and it will undoubtedly continue to do so long into the future. In differential geometry and differential equations, too, Griffiths has made many fundamental contributions. While his initial interest in these subjects was partly due to their immediate utility in algebraic and complex geometry and partly due to the influence of his postdoctoral mentor, Shiing-shen Chern, Griffiths developed a style and research program that were all his own and that have proved extraordinarily fertile.

Beginning with his beautiful 1974 article, “On Cartan's method of Lie groups and moving frames as applied to uniqueness and existence questions in differential geometry", Duke Mathematical Journal 41 (1974), 775-814, he brought to bear classical techniques on a variety of problems in real and complex geometry and laid out a program of applications to period mappings, Nevanlinna theory, integral geometry, and transcendental methods in algebraic geometry. This bore fruit in many papers over the years, 
particularly his 1978 paper with Chern, "Abel's theorem and webs", Jahresberichte der Deutschen Mathematiker Vereinigung 80 (1978), no. 1-2, 13110 , which presaged many of the recent developments in the recently active area of web geometry, and his 1979 paper with Joseph Harris, "Algebraic geometry and local differential geometry", Annales Scientifiques de l'École Normale Supérieure (4) 12 (1979), no. 3, 355-452.

His discovery of and investigations into what are now called the Griffiths infinitesimal period relations on period domains, which are of fundamental importance in moduli problems in algebraic geometry, stimulated his interest in overdetermined systems of differential equations. As a consequence, he led a revitalization of this subject in the 1980s in the form of exterior differential systems. Griffiths applied exterior differential systems to a number of different problems, not just in algebraic or complex differential geometry, but also to attack deep problems in modern differential geometry: rigidity of isometric embeddings in the overdetermined case and local existence of smooth solutions in the determined case in dimension 3, drawing deep results in hyperbolic PDEs (in collaborations with Berger, Bryant, and Yang); geometric formulations of integrability in the calculus of variations and in the geometry of Lax pairs; and treatises on the geometry of conservation laws and variational problems in elliptic, hyperbolic, and parabolic PDEs and exterior differential systems. All of these areas are currently seeing important developments that were stimulated by his work.

Phillip Griffiths' teaching career and research leadership, well measured by the numbers of mentored individuals who have gone on to stellar careers in mathematics and other disciplines, is simply astounding. His expository gifts and his nurturing of mathematical talent have not been reserved for his students alone. Not only has he been generous with his time, but he has written many classic expository papers and books, such as Principles of Algebraic Geometry with Joseph Harris, that have remained in print and inspired students of the subject since the 1960s.

A further fundamental characteristic of Phillip Griffiths is his extensive support of mathematics, both personally at the level of research and education and nationally and internationally through committees and boards he has chaired or served on. He has carried on a remarkable research career while serving eight years as Duke University's provost and twelve years as the director of the Institute for Advanced Study, and he currently chairs the Science Initiative Group, whose mission is assisting the development of mathematical training centers in the developing world. His example of service and leadership has inspired many in the mathematics community to emulate him to some degree, and our mathematical world is much the richer for it.

The Leroy P. Steele Prize for Lifetime Achievement is a further recognition to his dedication, generosity, and inspired leadership that surely fits the fiftieth anniversary of his receiving his Ph.D. from Princeton.

\section{Biographical Sketch}

Phillip A. Griffiths is Professor Emeritus in the School of Mathematics at the Institute for Advanced Study, where he was the director from 1991-2003 and a professor from 2004-2009. He was previously provost of Duke University. He has taught mathematics at Duke University, Harvard University, Princeton University, and the University of California Berkeley.

Dr. Griffiths was born in 1938 in Raleigh, North Carolina, and received his Bachelor of Science from Wake Forest University in 1959 and his Ph.D. from Princeton University in 1962. He was a Miller Fellow at UC Berkeley from 1962-1964 and again in 1976.

$\mathrm{He}$ is a member of the National Academy of Sciences and the American Philosophical Society and a foreign associate of the Accademia Nazionale dei Lincei, the World Academy of Sciences (TWAS), and the Indian Academy of Sciences. Dr. Griffiths was Chair of the Board on Mathematical Sciences at the National Research Council from 1986 to 1991, a member of the National Science Board from 1991 to 1996, Chair of the Committee on Science, Engineering and Public Policy at NAS/NAE/IOM from 1992 to 1999, Chair of the Program Committee for the International Congress of Mathematicians from 1995 to 1998, Secretary of the International Mathematical Union from 1999 to 2006, and co-chair of the Carnegie-IAS Commission on Mathematics and Science Education from 2007 to 2009. He received the Steele Prize for his paper "Periods of integrals on algebraic manifolds", Bulletin of the American Mathematical Society 7 (1970), 228-296, and more recently the Wolf and Brouwer Prizes.

Dr. Griffiths chairs the Science Initiative Group, an international team of scientists dedicated to building science and engineering capacity in developing countries through innovative programs, including the Millennium Science Initiative (MSI) and the Regional Initiative in Science and Education in Africa (RISE).

\section{Response from Phillip A. Griffiths}

It is a wonderful honor to receive the Leroy P. Steele Prize for Lifetime Achievement. I credit my high school math teacher, Lottie Wilson at the Georgia Military Academy, for sparking my interest in and love for mathematics. From my thesis advisor at Princeton University, Don Spencer, and my postdoctoral mentor at UC Berkeley, S. S. Chern, I learned how to think about math. From my students, collaborators and colleagues I have received far more than I could possibly have given. Finally, my wife, Taffy, our four children, and my 
colleagues in other activities have encouraged me to do what I love and have put up with a frequently distracted mathematician. To all of the above, and to the AMS and the selection committee for this award, I owe my deepest gratitude.

\section{About the Prize}

The Steele Prizes were established in 1970 in honor of George David Birkhoff, William Fogg Osgood, and William Caspar Graustein. Osgood was president of the AMS during 1905-1906, and Birkhoff served in that capacity during 1925-1926. The prizes are endowed under the terms of a bequest from Leroy P. Steele. Up to three prizes are awarded each year in the following categories: (1) Lifetime Achievement: for the cumulative influence of the total mathematical work of the recipient, high level of research over a period of time, particular influence on the development of a field, and influence on mathematics through Ph.D. students; (2) Mathematical Exposition: for a book or substantial survey or expository research paper; (3) Seminal Contribution to Research: for a paper, whether recent or not, that has proved to be of fundamental or lasting importance in its field or a model of important research. Each Steele Prize carries a cash award of US\$5,000.

The list of previous recipients of the Steele Prize may be found on the AMS website at http://www. ams.org/prizes-awards.

Beginning with the 1994 prize, there has been a five-year cycle of fields for the Seminal Contribution to Research Award. For the 2014 prize, the field was analysis. The Steele Prizes are awarded by the AMS Council acting on the recommendation of a selection committee.

For the 2014 awards, the members of the selection subcommittee for the Seminal Contribution to Research Award were Richard T. Durrett, Jeffrey C. Lagarias, Nikolai Makarov, Tomasz S. Mrowka (Chair), Andrei Okounkov, Gang Tian, LaiSang Young, and Efim I. Zelmanov. The members of the selection subcommittee for the Mathematical Exposition Award were Richard T. Durrett, Irene M. Gamba (Chair), Jeffrey C. Lagarias, Nikolai Makarov, Tomasz S. Mrowka, Andrei Okounkov, Gang Tian, Lai-Sang Young, and Efim I. Zelmanov. The members of the selection subcommittee for the Lifetime Achievement Prize were Richard T. Durrett, Irene M. Gamba (Chair), Jeffrey C. Lagarias, Nikolai Makarov, Tomasz S. Mrowka, Andrei Okounkov, Gang Tian, Lai-Sang Young, and Efim I. Zelmanov. -Elaine Kehoe

\section{Photo Credits}

The photo of Yuri Burago is courtesy of the St. Petersburg Division of the Steklov Math Institute; the painting of Dmitri Burago is by Dmitri Burago; photo of Luis Caffarelli is courtesy of ICES at the University of Texas Austin; photo of Phillip Griffiths is by Cliff Moore.

\section{Assistant Professor of Mathematical Finance}

\section{The Department of Mathematics} (www.math.ethz.ch) at ETH Zurich invites applications for the above-mentioned professorship. The research activities should be related to Mathematical Finance, for example computational and/or statistical and/or probabilistic aspects of quantitative finance and risk management. Duties of this position include an active participation in the teaching of courses for students of mathematics, natural sciences and engineering. The successful candidate holds a PhD degree and has demonstrated the ability to carry out independent research. It is expected to collaborate with colleagues and industry and to teach undergraduate level courses (German or English) and graduate level courses (English).

This assistant professorship has been established to promote the careers of younger scientists. The initial appointment is for four years with the possibility of renewal for an additional two-year period.

\section{Please apply online at}

www.facultyaffairs.ethz.ch

Applications should include a curriculum vitae, a list of publications, and a statement of your future research and teaching interests. The letter of application should be addressed to the President of ETH Zurich, Prof. Dr. Ralph Eichler. The closing date for applications is 15 April 2014. ETH Zurich is an equal opportunity and family friendly employer and is further responsive to the needs of dual career couples. In order to increase the number of women in leading academic positions, we specifically encourage women to apply. 